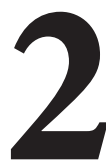

\title{
MOTIVACIÓN Y SATISFACCIÓN ACADÉMICA DE LOS ESTUDIANTES DE EDUCACIÓN: UNA VISIÓN INTERNACIONAL
}

\author{
(ACADEMIC MOTIVATION AND SATISFACTION AMONG STUDENTS OF \\ EDUCATION: AN INTERNATIONAL PERSPECTIVE)
}

Juan Llanes Ordóñez

Jorge Luis Méndez-Ulrich

Alejandra Montané López

Universitat de Barcelona

DOI: $10.5944 / e d u c X X 1.26491$

Cómo referenciar este artículo/How to reference this article:

Llanes Ordóñez, J.; Méndez-Ulrich, J.L. y Montané López, A. (2021). Motivación y satisfacción académica de los estudiantes de educación: una visión internacional. Educación XX1, 24(1), 4568, http://doi.org/10.5944/educXX1.26491

Llanes Ordóñez, J.; Méndez-Ulrich, J.L. \& Montané López, A. (2021). Academic motivation and satisfaction among students of education: an international perspective. Educación XX1, 24(1), 45-68, http://doi.org/10.5944/educXX1.26491

\section{RESUMEN}

El presente trabajo expone parte de los resultados del proyecto internacional To Tradition to Innovation in Teacher Training Institutions (TO-INN). En él participaron 13939 estudiantes universitarios de Europa y América Latina, que cursaban sus estudios en diferentes ámbitos de las Ciencias de la Educación. El objetivo del estudio fue trazar su perfil motivacional, así como detectar aquellas variables sociodemográficas y de satisfacción con la experiencia universitaria capaces de explicar y predecir la motivación académica. Para ello, se empleó un cuestionario compuesto por 33 ítems, que exploraba la dimensión social de estos estudiantes, indagando tanto variables sociodemográficas y de caracterización estudiantil, como de satisfacción con diferentes elementos de la experiencia universitaria, la 
participación universitaria y social y, finalmente, diferentes determinantes motivacionales intrínsecos y extrínsecos. Los cuestionarios fueron administrados online en 24 instituciones educativas de 3 países europeos y 7 de Latinoamérica. Los resultados sugieren un perfil sociodemográfico mayoritariamente femenino, con una media de edad próxima a los 25 años, de origen familiar socioeconómicamente medio o bajo, y que precisa de compaginar sus estudios con el trabajo. Respecto a los motivos para elegir la carrera, los intrínsecos asociados a la afiliación y el logro (ajuste a las propias capacidades, mejora del entorno social y placer por el estudio) son más determinantes para la elección de la carrera que los motivos extrínsecos, como el estatus social. Ambos tipos de motivación se asociaron de forma diferencial con diversos elementos de satisfacción académica. El modelo de regresión lineal mostró que las variables predictoras de la motivación fueron la edad, el nivel educativo y laboral de los progenitores, y el hecho de trabajar mientras estudiaban. Finalmente, se discuten las limitaciones del trabajo y se sugieren futuras líneas de investigación, especialmente en cuanto a la institución educativa como medio de promoción social, o el rol del género en la motivación académica.

\section{PALABRAS CLAVE}

Enseñanza superior, universidad, motivación para los estudios, satisfacción

\section{ABSTRACT}

This paper presents part of the results of the international project titled 'From Tradition to Innovation in Teacher Training Institutions (TOINN)', in which 13,939 European and Latin American university students taking Education Sciences participated. The objective of the study was to trace the students' motivational profiles and to detect which factors from their social backgrounds and academic satisfaction explained and predicted their academic motivation. To this end a 33-item questionnaire was used which explored the social dimensions of the students' backgrounds and experience, including socio-demographic variables, the type of student, their satisfaction with different aspects of their university experience, their social and university participation and a range of intrinsic and extrinsic motivation factors. The questionnaires were administered online at 24 educational institutions in 3 European countries and 7 in Latin America. Results suggest a mostly female socio-demographic profile, with an average age of around 25, of socio-economically medium or low family origin and needing to combine studying with working. Among their reasons 
for choosing the degree, intrinsic motives associated with identity and achievement (suitability to their aptitudes, desire for social mobility and the pleasure of studying for its own sake) carried more weight than extrinsic ones such as achieving enhanced social status. Both types of motivation were differentially associated with various elements of academic satisfaction. A linear regression model showed that the variables predicting motivation were the parents' age, education and type of employment and the fact of working while studying. Finally, the limitations of the study are discussed and future lines of research suggested, especially regarding the educational institution as a vehicle of social mobility and the role of gender in academic motivation.

\section{KEYWORDS}

Higher education, universities, student motivation, satisfaction

\section{INTRODUCCIÓN}

El estudio de los motivos para la elección de la carrera es un tema de interés creciente en la Educación Superior (Vázquez-Alonso y ManasseroMas, 2015). Según Abarca et al. (2012), la decisión de iniciar estudios universitarios implica un proceso de búsqueda y selección, en el que los estudiantes priorizan ciertos motivos, que pueden responder tanto a valoraciones personales y aspiraciones propias, como a factores externos, como las expectativas familiares o estereotipos sociales, vinculados a la distinción de motivos sociales clásica, postulada por McClelland (1961), entre motivos de logro, afiliación y poder. Por otra parte, de acuerdo con el modelo motivacional postulado por Deci y Ryan (2000), las razones de los estudiantes para elegir una carrera universitaria pueden estar fundamentadas en determinantes de carácter extrínseco relacionados con la promoción económica o el reconcomiendo social, o vinculadas a motivos intrínsecos (orientados al aprendizaje y la vida profesional), que en el ámbito académico se expresan en el disfrute por aprender o el deseo de logro vinculado al desarrollo personal y profesional exitoso, a partir de los estudios universitarios cursados (Cano-Celestino, 2008).

\section{¿Por qué y para qué cursar estudios superiores en Educación?}

Gámez y Marrero (2003), en su estudio comparativo entre estudiantes de carreras tan dispares como Psicología, Derecho y Biología, observaron cómo los motivos de elección no variaban significativamente en función 
del ámbito de conocimiento de que se tratase. Estos autores reportan que los motivos más importantes para los estudiantes (independientemente del área de conocimiento), son el interés por las relaciones interpersonales, la afiliación y prestigio y el poder, en línea de los motivos postulados en la teoría motivacional de McClelland (1961), y evaluados en diversos estudios como determinantes de la motivación para la elección de estudios (Gámez et al., 2015). Otros autores como García-Ripa et al. (2017) o Skatova y Ferguson (2014), sí que sugieren la existencia de diferencias en los perfiles motivacionales en función del ámbito de conocimiento.

En el caso concreto de las carreras de la Educación, diversos estudios han señalado el papel de los motivos intrínsecos, como el propio placer de enseñar y contribuir a la mejora social, como determinantes de su elección (Sultana y Wirtz, 1992; Yong, 1995). No obstante, los motivos extrínsecos, como obtener una fuente de ingresos segura o promocionar socialmente, también han mostrado ser de gran importancia a la hora de elegir este tipo de formaciones (García-Garduño y Organista, 2006). Para los estudiantes de Educación de origen socioeconómico bajo, la carrera universitaria representa un vehículo de movilidad social y promoción económica (Lent et al., 2016). Por su parte, Camina y Salvador (2007), también reportan que un elevado número de estudiantes de diversas disciplinas del Magisterio, habrían preferido haber accedido a otros estudios no relacionados con la Educación, lo que revela una preeminencia de la motivación extrínseca, y una preocupante falta de vocación por la profesión docente y pedagógica. En este sentido, existe abundante literatura que relaciona la motivación intrínseca con un mejor desempeño académico y laboral (Avendaño-Bravo y González-Urrutia, 2012), por lo que resulta imperativo conocer qué tipo de motivación influye de forma preeminente a los estudiantes de programas formativos relacionados con la Educación, para garantizar un óptimo desempeño profesional como posteriores profesionales educativos.

\section{Las características sociodemográficas como factor motivacional para la elección de la carrera}

Las características sociodemográficas (edad, nacionalidad, género, situación marital, etc.), pueden condicionar tanto el itinerario académico, como el modelo de universidad escogida. En este sentido, una edad de acceso temprana comporta itinerarios académicos lineales y sin pausas, mientras que, por el contrario, una edad de acceso tardía suele comportar la existencia de interrupciones (por motivos laborales, familiares, etc.), como señalan Figuera y Torrado (2015). Atendiendo a la flexibilidad de itinerarios en la universidad, cada vez es más frecuente encontrar en el aula estudiantes maduros (mayores de 25 años), así como otra gran diversidad de perfiles 
personales (estudiantes migrados, etc.). Esta realidad pone en relieve la necesidad de estudiar su trayectoria académica previa, para conocer sus motivos de acceso, y poder adaptar los planes formativos, e incidir en su satisfacción con la formación (Figuera et al., 2015).

Por otro lado, la distribución por géneros en las carreras universitarias está muy relacionada con la segregación selectiva, ya que algunas ramas de ciencias sociales o de la salud están más feminizadas que otras disciplinas (Torrents y Fachelli, 2015). De todos modos, la distribución por género varía en gran medida en función del contexto, según en el país donde se centre el estudio, y de los colectivos que acceden a la educación superior. No obstante, García-Ripa et al. (2017), inciden en que, en general, las carreras del ámbito social, y especialmente de la Educación, están profundamente feminizadas.

Las características socioeconómicas, a su vez, hacen referencia al background familiar del cual proceden los estudiantes que acceden a la universidad, y que en muchas ocasiones determina el abandono o persistencia en la propia formación. Los datos obtenidos en distintos estudios señalan la importancia de este factor, tanto por el nivel adquisitivo, como por el capital formativo y cultural (Troiano et al., 2017). Los estudios en el contexto europeo han constatado que existe una sobrerrepresentación de los estudiantes procedentes de núcleos familiares de clase social alta con estudios superiores y un perfil ocupacional también de alta cualificación (Ariño y Síntes, 2016; Cabrera et al., 2014; Martínez-García, 2017). Ahora bien, la literatura también ha señalado que las formaciones del ámbito de las ciencias sociales son las que recogen un mayor número de estudiantes procedentes de familias con baja o media cualificación, y de clase socioeconómica baja o media, que intentan acceder a estudios (que ellos consideran más accesibles), buscando su promoción social y cultural (Martínez-García, 2017). No obstante, no existen estudios sistemáticos sobre las condiciones de vida y estudio del colectivo en cuestión en el contexto iberoamericano, desde la óptica de equidad participativa, aspecto que es de especial relevancia abordar (Méndez-Ulrich et al., 2019).

\section{Motivación y satisfacción con la experiencia universitaria}

Tinto (2017), en su modelo de integración y éxito académico, evidencia que la motivación es un factor fundamental para explicar la satisfacción con la elección de los estudios y las decisiones de continuidad en la carrera universitaria. Además, estos motivos pueden condicionar el proyecto formativo y profesional de continuidad tras la graduación (Dorio, 2017). Este proceso de integración, mediado por la propia satisfacción 
académica, responde a factores personales, contextuales e institucionales. Los factores personales se vinculan a la propia capacidad, motivación inicial y satisfacción de logro de resultados posteriores (Maurer y Chapman, 2017). Los factores contextuales (docentes, compañeros, clima de clase, estimulación académica...), por su parte, refuerzan el proceso del estudiante por su itinerario universitario (Griffioen et al., 2018). Por último, los factores institucionales refuerzan todo el apoyo que se deriva de las instancias universitarias, tales como la secretaría, los servicios de orientación, el plan de estudio, etc. (Weerasinghe et al., 2018). Todas estas variables pueden decantar la balanza hacia la persistencia o el abandono académico y, por ende, la calidad de la institución de referencia y del propio sistema educativo (Chandra et al., 2018).

En este sentido, investigaciones a nivel de grado y postgrado, como la llevada a cabo por Figuera et al. (2018), apuntan a la relevancia del ajuste entre las motivaciones iniciales, las estrategias de aprendizaje, la satisfacción académica; y la posterior graduación y adecuación del proyecto profesional a la carrera escogida. Por otra parte, estas autoras constataron que aquellas personas con mayor edad y experiencia previa con el mundo del trabajo tenían más clara su elección universitaria, hecho que condicionaba la satisfacción con sus estudios. Si nos ceñimos a los títulos de grado, el inicio de la carrera universitaria es el momento en que los estudiantes concentran más dudas en relación con su elección. No obstante, continúa inexplorado el rol diferencial de la motivación intrínseca y extrínseca en la satisfacción con la experiencia universitaria de los estudiantes de educación superior del ámbito de la Educación.

\section{MÉTODO}

\section{Objetivo}

En este trabajo, se presenta un extracto de los resultados del proyecto From Tradition to Innovation in Teacher Training Institutions (TO-INN), del programa Erasmus+. Este proyecto parte de una visión sistémica de la calidad en Educación Superior, en la que la inclusión, el acceso, la supervivencia y el éxito académico de los estudiantes juegan un papel relevante. La perspectiva del proyecto acepta que el proceso de masificación vivido en las universidades, a través de los procesos de democratización, convive con las desigualdades tanto en el acceso, como en la permanencia y los resultados académicos. En esta línea, se realizó un estudio cuantitativo, empleando la metodología de cuestionario, cuyos objetivos fueron: 
a) Identificar las características sociodemográficas de los estudiantes de programas formativos vinculados a las Ciencias de la Educación y sus condiciones de vida.

b) Determinar el perfil motivacional y el nivel de satisfacción con la experiencia universitaria de los estudiantes de Educación.

c) Establecer la relación entre las características sociodemográficas de los estudiantes, los motivos para la elección de la carrera y la satisfacción con la experiencia universitaria.

\section{Participantes}

En el proyecto participaron 21 instituciones universitarias (más un centro o escuela adscrita a cada universidad), de 13 países de Europa $(n=5)$ y América Latina $(n=8)$. Para su inclusión en el presente estudio, era necesario que al menos 50 estudiantes de cada institución contestaran al cuestionario, condición que no se dio en las instituciones de tres países. Por tanto, finalmente en este artículo se incluyen los datos de 13939 estudiantes de grados y postgrados del ámbito de la Educación de 24 instituciones de España, Portugal, Francia, Colombia, Argentina, Ecuador, Honduras, Bolivia, Paraguay y México. Todos estos centros eran de titularidad pública, excepto dos centros de Bolivia, y uno en Portugal, que eran de titularidad privada. La población total de los programas formativos evaluados en el estudio era de 30902 estudiantes. Por tanto, nuestra muestra representó un $45.11 \%$ del total, con un error muestral del 0.61. De estos participantes, 9279 $(66.6 \%)$ fueron mujeres con una media de edad de 24.44 años (D.T $=7.39)$, y $4660(33.4 \%)$ hombres con una edad promedio de 24.95 años (D.T. $=7.57)$. De estos, $1712(12.28 \%)$ pertenecían a instituciones Europas, mientras que el resto cursaban sus estudios en centros de América Latina.

\section{Instrumento}

Con el fin de analizar las variables que componen la dimensión social de los estudiantes universitarios que cursan estudios en Educación, el equipo coordinador creó un cuestionario compuesto por 33 ítems que contenían preguntas relativas a cuatro dimensiones del estudio: 1) datos sociodemográficos, edad, género, nivel de estudios y ocupación de los progenitores (categoriales); 2) datos académicos, estudios, vía de acceso, ayudas (categoriales), y satisfacción con la experiencia universitaria (general, compañeros, contenidos académicos, profesorado, servicios de gestión universitaria, y de orientación); 3) vida social y participación 
(situación residencial y laboral, instituciones de participación ciudadana (categoriales); y 4) ámbito motivacional, intrínsecos como la adquisición de conocimientos, el ajuste a las propias capacidades, o la contribución a la mejora de la sociedad; y extrínsecos: salario, posición social, o presión familiar para cursar estos estudios. Estos motivos, fueron además categorizados en motivos de afiliación, logro y poder. Tanto la satisfacción como la motivación estaban constituidas por 6 ítems escalares de tipo Likert, con un rango de 1 a 5 (de 1 totalmente en desacuerdo, a 5, totalmente de acuerdo).

Una vez creado el cuestionario, y tras sucesivas revisiones, mediante el consenso del conjunto de socios se realizó una prueba piloto en la que participaron 9 universidades, aportando un total de 299 respuestas. Fruto de este piloto, se determinó que su tiempo de aplicación era pertinente. Se realizaron diversas adaptaciones en cuanto a la redacción de aquellos ítems escalares que mostraron dificultades de comprensión, o la supresión o inclusión de la opción "otros", en los ítems de respuesta múltiple. La versión completa del cuestionario puede consultarse en Montané et al. (2019).

\section{PROCEDIMIENTO}

Las instituciones participantes distribuyeron entre sus estudiantes una versión online del cuestionario creada a través de la plataforma Survey Monkey ${ }^{\circledR}$, a partir de diversos medios electrónicos entre junio de 2018 y febrero de 2019.

Para la explotación estadística se empleó el software IBM Statistical Package for Social Sciences (SPSS ${ }^{\circledR}$ para Windows, versión 22). En primer lugar, se procedió a la depuración de la matriz de datos, eliminando aquellos casos duplicados, incompletos (menos del 60\%), y suprimiendo los casos provenientes de instituciones con un número de respuestas insuficiente. De este modo, en total se depuraron 881 casos. A continuación, se computaron las puntuaciones globales de motivación intrínseca y extrínseca, y de la escala de satisfacción con la experiencia universitaria, a partir del sumatorio de los ítems de cada escala. Para los análisis descriptivos de las características sociodemográficas se utilizaron la media y la deviación típica, en el caso de las variables de intervalo, y los porcentajes para las variables categoriales. La estadística inferencial consistió en la obtención de correlaciones bivariadas entre las puntuaciones de las variables de tipo escalar y en el análisis de la varianza (oneway ANOVA), para la comparación de medias en las escalas, en función de las diversas variables sociodemográficas. Para detectar aquellas variables sociodemográficas predictoras de la motivación intrínseca y extrínseca de los participantes, se realizaron dos modelos de 
regresión lineal multivariado (método de pasos sucesivos), en el que la motivación intrínseca y extrínseca fueron las variables dependientes, y las variables sociodemográficas fueron las variables independientes. A tal efecto, las variables categoriales fueron computadas como ordinales, y codificadas numéricamente. Finalmente, para poder establecer diferencias estadísticamente significativas entre las puntuaciones medias en los indicadores de motivación y satisfacción, en función de variables ordinales como la cantidad de horas dedicadas al trabajo remunerado o el curso que se estaba estudiando en el momento de contestar el cuestionario, se empleó también el análisis de la varianza (oneway ANOVA), con estimación de contrastes Post-hoc, mediante el método Bonferroni.

\section{RESULTADOS}

\section{Perfil sociodemográfico}

Como se indica en el apartado de participantes, la distribución muestral por género y edad obtenida en nuestro estudio estuvo compuesta por un $66.6 \%$ de mujeres con una media de edad de 24.44 años (D.T. $=7.39$ ), y un $33.4 \%$ de hombres, cuya media de edad fue de 24.95 años (D.T. $=7.57$ ). De estos, el $57.7 \%$ residían con sus padres, el $13.1 \%$ con su pareja, y un $14.4 \%$ vivían en una vivienda alquilada junto con otras personas. Un $14.3 \%$ de personas vivían en viviendas propias o alquiladas por ellas mismas. Resulta destacable que únicamente un $0.5 \%$ de las personas encuestadas declararan vivir en una residencia universitaria.

En cuanto a la situación laboral, el 51.5\% no realizaba ninguna actividad remunerada en el momento de contestar el cuestionario. Un 29.5\% tenía un trabajo relacionado con el ámbito profesional de la Educación, mientras que el 19\% restante trabajaba en un ámbito completamente diferente. Un $32.4 \%$ de la muestra declaró tener que atender de forma simultánea estudios y personas dependientes. Únicamente un $4.6 \%$ de las personas encuestadas tenía reconocida alguna situación de diversidad funcional (física, cognitiva o sensorial).

Respecto a las variables sociodemográficas relacionadas con la familia, tal y como se observa en la Figura 1, tanto en el caso de la madre como del padre, el nivel educativo alcanzado de forma más frecuente era el de estudios básicos o secundarios. En el caso de las madres la suma de estos tipos de estudios representaba el $62.7 \%$ del total, y el $61.9 \%$ en el caso de los padres. Por otra parte, era infrecuente el hecho de que estos no contaran con ningún tipo de formación. No obstante, también fue llamativo el escaso porcentaje de progenitores con estudios universitarios. 
Figura 1

Nivel de estudios de los progenitores en porcentajes

sobre el total de la muestra.

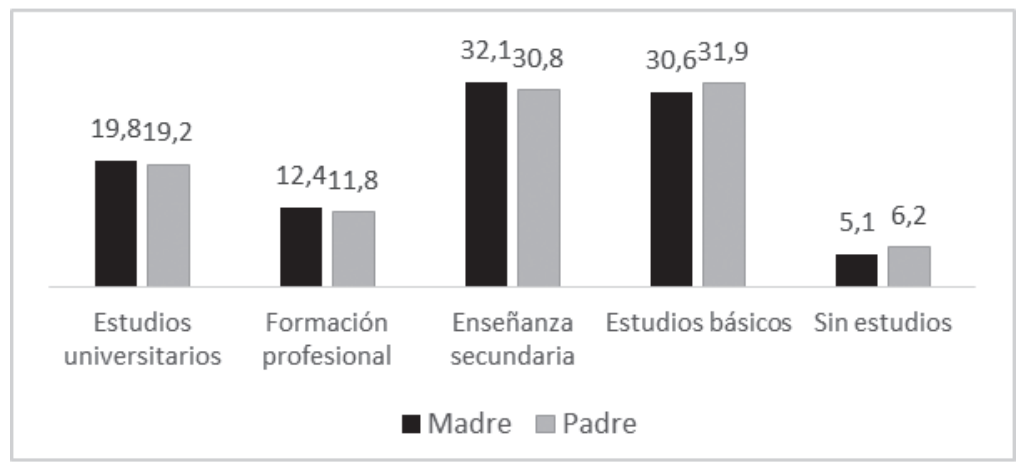

Fuente: Elaboración propia

En relación con la ocupación de los padres, tal como se resume en la Figura 2, en el caso del padre, la ocupación laboral más frecuente eran los trabajos relacionados con cargos intermedios (45.9\% de los casos), mientras el $55.8 \%$ de las madres desarrollaban trabajos poco cualificados. Resulta interesante observar cómo, a medida que el nivel ocupacional aumenta, la presencia de la mujer disminuye, y viceversa $\left(X^{2}=2844.00, \mathrm{gl}=9, \mathrm{p}<.005\right)$. Esto resultó especialmente evidente en relación con el trabajo doméstico remunerado, ocupación que desarrollaban un $11.2 \%$ de las madres, y un $3.6 \%$ de los padres.

\section{Figura 2}

Ocupación de los progenitores en porcentajes sobre el total de la muestra.

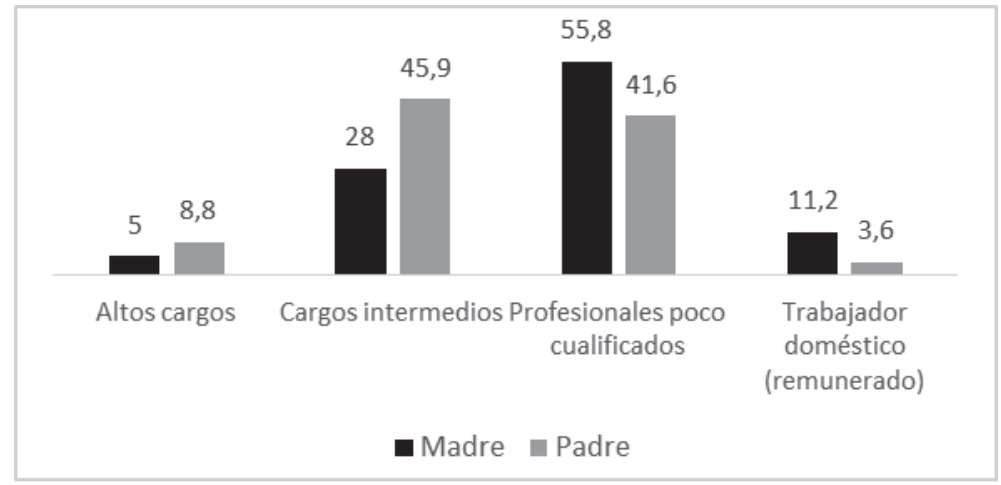

Fuente: Elaboración propia 


\section{Perfil motivacional}

En cuanto al tipo de motivación más frecuente en los estudiantes, la mayor parte mostró un perfil en el que el sumatorio de los motivos intrínsecos $($ media $=13.59 ; \mathrm{D} . \mathrm{T}=2.08)$ resultaron ser más elevados que los extrínsecos (media $=8.20 ;$ D.T.= 3.05); siendo esta diferencia estadísticamente significativa $(\mathrm{t}=169.11 ; \mathrm{p}<.005)$. A un nivel micro analítico (ítem a ítem), como se observa en la Tabla 1 (que muestra de forma jerarquizada los factores motivacionales más relevantes), los tres determinantes motivacionales que obtuvieron mayor puntuación fueron todos de tipo intrínseco, siendo los tres ítems que evaluaban la motivación extrínseca los que obtuvieron una puntuación más baja.

Tabla 1

Media y desviación típica en los ítems de la escala de motivación

\begin{tabular}{lccc}
\hline \multicolumn{1}{c}{ Ítem } & Motivación & Media & D.T. \\
\hline $\begin{array}{l}\text { Cultivarme y enriquecer mis conocimientos o } \\
\text { aprendizajes }\end{array}$ & $\begin{array}{c}\text { Intrínseca } \\
\text { (logro) }\end{array}$ & 4.62 & 0.77 \\
$\begin{array}{l}\text { Poder contribuir a la mejora de la sociedad } \\
\text { en general y/o de mi comunidad más próxima }\end{array}$ & $\begin{array}{c}\text { Intrínseca } \\
\text { (afiliación) }\end{array}$ & 4.60 & 0.80 \\
Se corresponde con mis aptitudes & $\begin{array}{c}\text { Intrínseca } \\
\text { (logro) }\end{array}$ & 4.37 & 0.89 \\
$\begin{array}{l}\text { Asegurarme unos ingresos y/o alcanzar una } \\
\text { mejor posición social }\end{array}$ & $\begin{array}{c}\text { Extrínseca } \\
\text { (poder) }\end{array}$ & 3.78 & 1.30 \\
$\begin{array}{l}\text { La elegí aunque no era mi primera opción de } \\
\text { preferencia }\end{array}$ & $\begin{array}{c}\text { Extrínseca } \\
\text { (logro) }\end{array}$ & 2.66 & 1.65 \\
Por tradición familiar & $\begin{array}{c}\text { Extrínseca } \\
\text { (afiliación) }\end{array}$ & 1.77 & 1.33 \\
\hline
\end{tabular}

Fuente: Elaboración propia

Estos resultados sugieren que los factores motivacionales de los estudiantes de carreras de la Educación tuvieron que ver mayoritariamente con: a) el logro vinculado con el placer por el aprendizaje y el desarrollo de las propias capacidades y aptitudes, y b) los motivos afiliativos relacionados con la mejora del entorno social y la comunidad más próxima. Por otra parte, entre los determinantes motivacionales extrínsecos, la puntuación más elevada fue la del ítem relacionado con la adquisición de un estatus social y económico mejor al de procedencia, siendo prácticamente residual la influencia de la presión del entorno familiar para estudiar este tipo de carreras. Por su parte, el análisis de correlaciones bivariadas indicó que el género es una variable relevante para determinar el tipo de motivación preeminente en la elección de estudios superiores en Educación, siendo 
superior en las mujeres que en los hombres tanto en el caso de la motivación intrínseca $(\mathrm{F}=12.43 ; \mathrm{p}<.001)$, como de la extrínseca $(\mathrm{F}=57.97 ; \mathrm{p}<.001)$,

\section{Motivación y satisfacción con la experiencia universitaria}

El análisis de correlaciones bivariadas mostró una asociación significativa al nivel .01 (bilateral), entre las puntuaciones globales de motivación intrínseca y extrínseca con todos los indicadores de satisfacción con la experiencia universitaria. No obstante, como se puede observar en la Tabla 2, los coeficientes de correlación entre dichos indicadores y las puntuaciones de motivación intrínseca eran sistemáticamente superiores a las correlaciones con la puntuación de motivación extrínseca, lo que indica una mayor satisfacción con la experiencia universitaria en aquellos estudiantes en los que la motivación intrínseca era la preeminente.

Tabla 2

Correlaciones entre las escalas motivacionales y los indicadores de satisfacción con la experiencia universitaria

\begin{tabular}{|c|c|c|c|c|c|c|c|}
\hline & 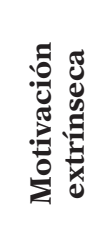 & 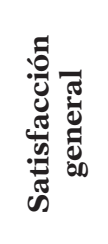 & 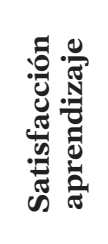 & 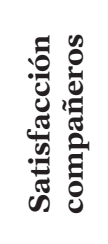 & 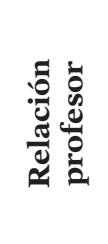 & 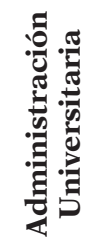 & 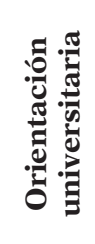 \\
\hline $\begin{array}{l}\text { Motivación } \\
\text { Intrínseca }\end{array}$ & $\begin{array}{c}.037 \\
<.001\end{array}$ & $\begin{array}{c}.373 \\
<.001\end{array}$ & $\begin{array}{c}.429 \\
<.001\end{array}$ & $\begin{array}{c}.279 \\
<.001\end{array}$ & $\begin{array}{c}.346 \\
<.001\end{array}$ & $\begin{array}{c}.037 \\
<.001\end{array}$ & $\begin{array}{l}.314 \\
<.001\end{array}$ \\
\hline $\begin{array}{l}\text { Motivación } \\
\text { extrínseca }\end{array}$ & & $\begin{array}{l}.113 \\
<.001\end{array}$ & $\begin{array}{l}.084 \\
<.001\end{array}$ & $\begin{array}{l}.100 \\
<.001\end{array}$ & $\begin{array}{l}.116 \\
<.001\end{array}$ & $\begin{array}{l}.151 \\
<.001\end{array}$ & $\begin{array}{l}.165 \\
<.001\end{array}$ \\
\hline $\begin{array}{l}\text { Satisfacción } \\
\text { general }\end{array}$ & & & $\begin{array}{c}.598 \\
<.001\end{array}$ & $\begin{array}{c}.439 \\
<.001\end{array}$ & $\begin{array}{c}.534 \\
<.001\end{array}$ & $\begin{array}{c}.084 \\
<.001\end{array}$ & $\begin{array}{l}.532 \\
<.001\end{array}$ \\
\hline $\begin{array}{l}\text { Satisfacción } \\
\text { aprendizaje }\end{array}$ & & & & $\begin{array}{l}.437 \\
<.001\end{array}$ & $\begin{array}{l}.598 \\
<.001\end{array}$ & $\begin{array}{l}.461 \\
<.001\end{array}$ & $\begin{array}{l}.536 \\
<.001\end{array}$ \\
\hline $\begin{array}{l}\text { Satisfacción } \\
\text { compañeros }\end{array}$ & & & & & $\begin{array}{c}.512 \\
<.001\end{array}$ & $\begin{array}{c}.414 \\
<.001\end{array}$ & $\begin{array}{l}.406 \\
<.001\end{array}$ \\
\hline $\begin{array}{l}\text { Relación } \\
\text { profesorado }\end{array}$ & & & & & & $\begin{array}{l}.564 \\
<.001\end{array}$ & $\begin{array}{l}.578 \\
<.001\end{array}$ \\
\hline $\begin{array}{l}\text { Admin. Uni- } \\
\text { versitaria }\end{array}$ & & & & & & & $\begin{array}{l}.727 \\
<.001\end{array}$ \\
\hline
\end{tabular}

Fuente: Elaboración propia 
Por otra parte, la motivación intrínseca se asoció con mayor intensidad (aunque de forma moderada) con aquellos indicadores de satisfacción relacionados con la experiencia de aprendizaje y la relación con el profesorado, mientras que la motivación extrínseca correlacionó con mayor potencia con el indicador que podría considerarse como más instrumental para la obtención del título y la futura inserción laboral: la satisfacción con la orientación universitaria recibida. La satisfacción general con la experiencia universitaria también correlacionó con mayor intensidad con la motivación intrínseca que con la extrínseca.

Finalmente, para detectar un posible efecto sobre la satisfacción con la experiencia universitaria, se introdujo la variable curso en la comparación de medias en los ítems de satisfacción entre estudiantes de $1^{\circ}$ a $4^{\circ}$ curso, incluyendo los estudiantes de postgrado. Los resultados indicaron una tendencia global al decaimiento de los indicadores de satisfacción a lo largo de la carrera, si bien en los estudiantes de postgrado aumentaba nuevamente, excepto para la satisfacción con la administración universitaria y la orientación recibida por la institución, como se observa en la Tabla 3.

Tabla 3

ANOVA de los indicadores de satisfacción en función de la veteranía en el programa

\begin{tabular}{cccccccc}
\hline & & $\begin{array}{c}\text { Satis. } \\
\text { general }\end{array}$ & $\begin{array}{c}\text { Satis. } \\
\text { aprendizaje }\end{array}$ & $\begin{array}{c}\text { Satis. } \\
\text { compañeros }\end{array}$ & $\begin{array}{c}\text { Relación } \\
\text { profesor. }\end{array}$ & $\begin{array}{c}\text { Admin. } \\
\text { universit. }\end{array}$ & $\begin{array}{c}\text { Servicios } \\
\text { orientación }\end{array}$ \\
\hline Promedio & Media & 4.14 & 4.35 & 4.13 & 4.19 & 3.83 & 3.86 \\
& D.T. & .97 & .91 & 1.03 & .99 & 1.20 & 1.20 \\
$1^{\circ}$ & Media & 4.19 & 4.38 & 4.19 & 4.25 & 4.09 & 4.06 \\
& D.T. & .94 & .90 & 1.01 & .93 & 1.07 & 1.10 \\
$2^{\circ}$ & Media & 4.14 & 4.38 & 4.13 & 4.20 & 3.81 & 3.87 \\
& D.T. & .98 & .90 & 1.03 & .94 & 1.20 & 1.21 \\
$3^{\circ}$ & Media & 4.11 & 4.35 & 4.08 & 4.14 & 3.72 & 3.77 \\
& D.T. & .95 & .89 & 1.02 & .93 & 1.19 & 1.20 \\
$4^{\circ}$ & Media & 4.02 & 4.21 & 4.04 & 4.06 & 3.51 & 3.59 \\
& D.T. & .99 & .98 & 1.04 & .99 & 1.26 & 1.27 \\
\multirow{2}{*}{ Postgrado } & Media & 4.21 & 4.40 & 4.17 & 4.23 & 3.82 & 3.87 \\
& D.T. & .96 & .87 & 1.04 & .95 & 1.24 & 1.23 \\
\hline
\end{tabular}

Fuente: Elaboración propia 
La Tabla 4 muestra la significación estadística de las diferencias de medias de la tabla anterior, obtenidas mediante el análisis de contrastes Post-hoc (método Bonferroni). Para una mayor claridad expositiva, se muestra únicamente la significación (en negrita) entre las diferencias de medias entre los estudiantes de primer curso y el resto.

Tabla 4

Contrastes Post-Hoc de los indicadores de satisfacción en función del curso

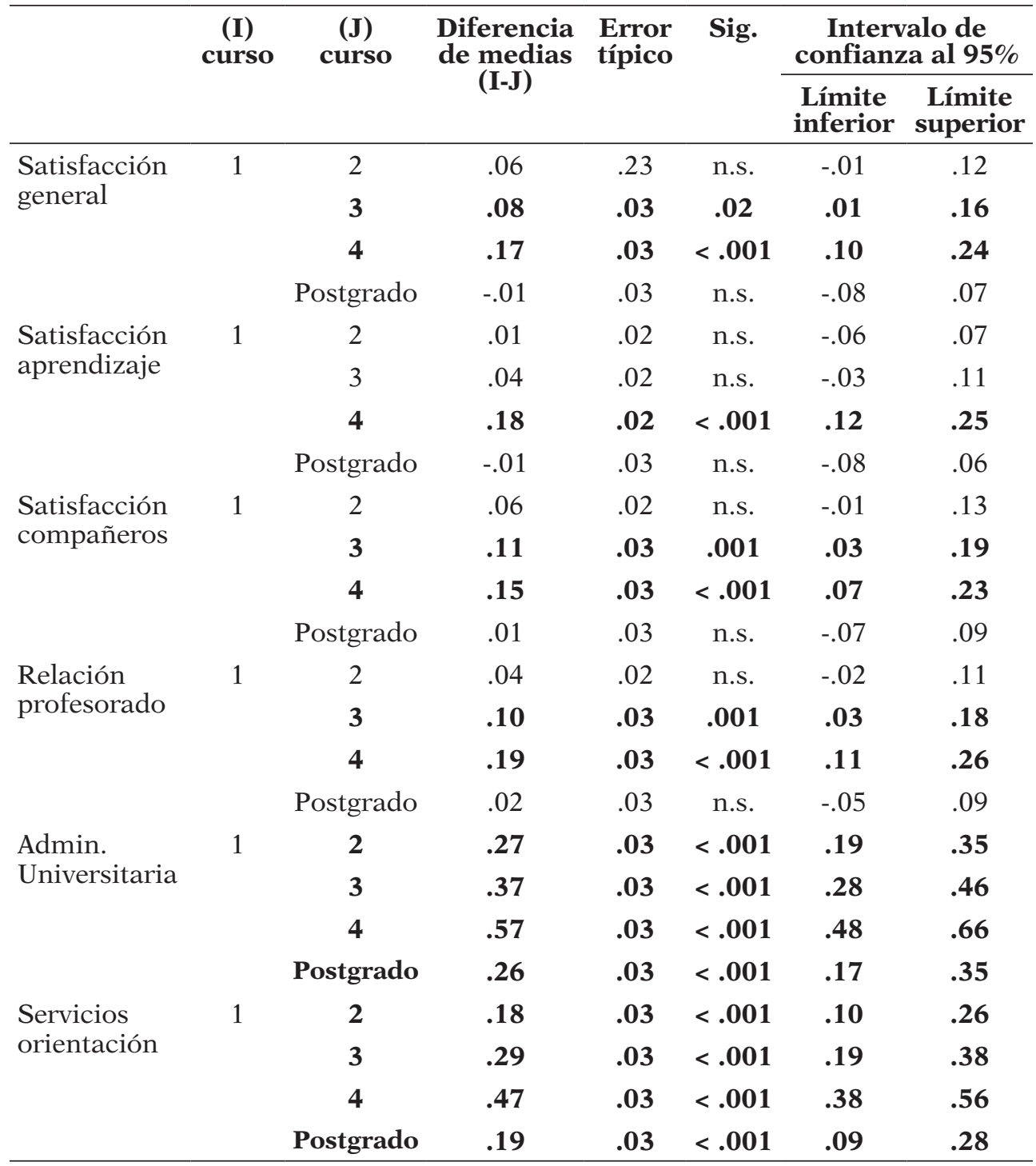

Fuente: Elaboración propia 
En cuanto a la relación entre el trabajo remunerado y la motivación, la Tabla 5 muestra una tendencia diferencial, en cuanto a los niveles de motivación intrínsecos como extrínsecos, en función de la cantidad de horas dedicadas al trabajo remunerado. En el caso de los estudiantes orientados por motivos intrínsecos, esta aumenta a medida que se incrementan las horas dedicadas al trabajo. No obstante, la motivación extrínseca disminuye a medida que aumentan las horas dedicadas al trabajo, excepto en el caso de los estudiantes que han de dedicar al trabajo remunerado más de 30 horas semanales.

Tabla 5

Medias de motivación intrínseca y extrínseca en función de la cantidad de horas dedicadas al trabajo remunerado

\begin{tabular}{lcccccc}
\hline & \multicolumn{3}{c}{ Motivación intrínseca } & \multicolumn{3}{c}{ Motivación extrínseca } \\
\multicolumn{1}{c}{ Horas trabajadas } & N & Media & D.T. & N & Media & D.T. \\
\hline Sin trabajo remunerado & 6661 & 13.50 & 2.14 & 6661 & 8.37 & 3.07 \\
De 1 a 15 horas semanales & 2644 & 13.54 & 2.08 & 2644 & 7.92 & 3.02 \\
De 15 a 29 horas semanales & 1608 & 13.64 & 2.03 & 1608 & 7.93 & 2.97 \\
Más de 30 horas semanales & 2066 & 13.89 & 1.90 & 2066 & 8.24 & 3.03 \\
Total & 12979 & 13.59 & 2.08 & 12979 & 8.20 & 3.05 \\
\hline
\end{tabular}

Fuente: Elaboración propia

Los análisis de contraste Post-hoc, revelaron que la única diferencia estadísticamente significativa entre la puntuación de motivación intrínseca y las horas dedicadas al trabajo remunerado fue la existente entre el hecho de no trabajar durante los estudios, y trabajar más de 30 horas semanales $(\mathrm{p}<.001)$, mientras que, en el resto de los casos las diferencias de medias fueron inapreciables. En relación con la motivación extrínseca, los datos sugieren que existe una diferencia estadísticamente significativa entre el hecho de no trabajar y trabajar menos de 15 horas $(\mathrm{p}<.001)$, y entre 15 y 29 horas $(\mathrm{p}<.001)$, pero no entre no trabajar y hacerlo más de 30 horas semanales.

\section{Variables predictoras de la motivación intrínseca y extrínseca}

Para poder establecer qué variables sociodemográficas otorgan una mayor potencia explicativa a la motivación, tanto intrínseca como extrínseca, se llevaron a cabo dos modelos de regresión lineal múltiple mediante el método de pasos sucesivos (stepwise). En cuanto a la motivación intrínseca, (Tabla 6). El modelo reportó que las variables predictoras de este tipo de 
motivación fueron: ser mujer, el nivel de estudios de la madre y del padre (a mayor cualificación académica, mayor motivación), la edad (a mayor edad, más motivación), y el número de horas trabajadas a la semana. Por otra parte, el modelo sugiere que pertenecer a una comunidad indígena afecta de forma negativa a la motivación intrínseca.

Como se muestra en la Tabla 7 , algunas variables predictoras de la motivación intrínseca también resultaron predictoras de la motivación extrínseca. A las variables predictoras de la motivación extrínseca se une el nivel laboral de ambos progenitores. A este respecto, el modelo sugirió que, según aumenta la cualificación profesional del padre, mayor motivación extrínseca; mientras que ésta aumenta a menor cualificación de la madre. En cuanto al género, el modelo sugiere que los hombres tienen mayores niveles de motivación extrínseca que las mujeres. Finalmente, también se observa que este tipo de motivación disminuye a medida que aumentan las horas de trabajo que los estudiantes dedican al trabajo de forma simultánea a sus estudios. El coeficiente de determinación, tanto del modelo predictivo de la motivación intrínseca como de la motivación extrínseca fue: $r=.14$; $\mathrm{R}^{2}$ corregido $=21 ; \mathrm{y} \mathrm{R}^{2}$ ajustado $=.020$, lo que sugiere que las variables seleccionadas fueron predictoras del $20 \%$ de la variabilidad de la puntuación total de motivación, tanto intrínseca como extrínseca, evaluadas mediante el cuestionario del estudio.

Tabla 6

Modelo de regresión lineal predictivo de la motivación intrínseca

\begin{tabular}{|c|c|c|c|c|c|}
\hline \multirow[b]{2}{*}{ Modelo } & \multicolumn{2}{|c|}{$\begin{array}{l}\text { Coeficientes no } \\
\text { estandarizados }\end{array}$} & \multirow{2}{*}{$\begin{array}{c}\text { Coeficientes } \\
\text { estandarizados } \\
\text { Beta }\end{array}$} & \multirow[b]{2}{*}{$\mathbf{t}$} & \multirow[b]{2}{*}{ Sig. } \\
\hline & B & $\begin{array}{l}\text { Error } \\
\text { tip. }\end{array}$ & & & \\
\hline Constante & 12.40 & .16 & & 76.14 & $<.001$ \\
\hline $\begin{array}{l}\text { Nivel de estudios de la } \\
\text { madre }\end{array}$ & .12 & .03 & .07 & 4.47 & $<.001$ \\
\hline Edad & .02 & .01 & .06 & 4.41 & $<.001$ \\
\hline $\begin{array}{l}\text { Nivel de estudios del } \\
\text { padre }\end{array}$ & .09 & .03 & 0.5 & 3.35 & .001 \\
\hline Género femenino & .15 & .05 & .03 & 2.99 & .003 \\
\hline $\begin{array}{l}\text { Horas dedicadas al } \\
\text { trabajo remunerado }\end{array}$ & .07 & .02 & .04 & 2.84 & .005 \\
\hline $\begin{array}{l}\text { Pertenecer a una } \\
\text { comunidad indígena }\end{array}$ & -.21 & .09 & .03 & -2.35 & .019 \\
\hline
\end{tabular}

Fuente: Elaboración propia 
Tabla 7

Modelo de regresión lineal predictivo de la motivación extrínseca

\begin{tabular}{lcccccc}
\hline \multicolumn{1}{c}{ Modelo } & $\begin{array}{c}\text { Coeficientes no } \\
\text { estandarizados }\end{array}$ & $\begin{array}{c}\text { Coeficientes } \\
\text { estandarizados }\end{array}$ & & \\
& B & $\begin{array}{c}\text { Error } \\
\text { tip. }\end{array}$ & Beta & t & Sig. \\
\hline Constante & 9.479 & .28 & & 33.85 & $<.001$ \\
Género femenino & -.55 & .08 & .09 & -7.36 & $<.001$ \\
$\begin{array}{l}\text { Pertenencia a pueblo } \\
\text { indígena }\end{array}$ & -.87 & .13 & .08 & -6.74 & $<.001$ \\
Edad & .04 & .01 & .08 & 6.63 & $<.001$ \\
Horas de trabajo semanales & -.18 & .04 & .06 & -5.12 & $<.001$ \\
Nivel ocupacional del padre & .21 & .06 & .05 & 3.69 & $<.001$ \\
Nivel ocupacional de la & -.19 & .06 & .04 & -3.41 & .001 \\
madre & & & & & & \\
\hline
\end{tabular}

Fuente: Elaboración propia

\section{DISCUSIÓN Y CONCLUSIONES}

Los resultados del estudio sugieren que existe un perfil sociodemográfico de estudiantes de los programas formativos del ámbito de la Educación coherente con la literatura previa (García-Ripa et al., 2017; Figuera y Torrado, 2015); tratándose en su mayor parte de mujeres con una media de edad de 24.4 años, proveniente de familias con un nivel socioeconómico y educativo medio o bajo, con una ocupación laboral de nivel medio, y que en su mayoría reside o en el domicilio familiar o compartiendo vivienda (Torrents y Fachelli, 2015). Es importante destacar que un porcentaje prácticamente residual de la muestra pertenecía a algún población indígena o pueblo originario, o presentaba alguna discapacidad, lo que apunta a una preocupante falta de equidad en el acceso a la Educación Superior. Además, el perfil sociodemográfico de los encuestados resulta congruente, a priori, con la hipótesis del ascensor social, que sostiene que la promoción social es uno de los motivos principales para la elección de ir a la universidad (Torrents y Fachelli, 2015; Troiano et al., 2017).

En este sentido, el deseo de mejora del contexto social, que es un claro reflejo del motivo de afiliación postulado por McClelland (1961), fue el motivo que obtuvo una mayor puntuación en nuestro estudio, en sintonía con los resultados reportados por Ariño y Síntes (2016) y Cabrera et al. (2014). Aunque todos los motivos intrínsecos fueron más relevantes 
que los extrínsecos en el acceso, es interesante señalar que dentro de los extrínsecos el más determinante fue el vinculado a la promoción económica y social individual, vinculado a la expectativa de obtener prestigio y una mejor posición social, como afirma Martínez-García (2017). En cuanto a la relación entre el hecho de trabajar durante los estudios y los indicadores de motivación, nuestros resultados sugieren que, especialmente la motivación extrínseca se ve afectada por ese hecho, en el sentido que, a más horas trabajadas, por lo general, se observa un menor nivel de este tipo de motivación. Esto podría explicarse por el hecho de que los estudiantes orientados a motivos económicos o prestigio social serían más vulnerables a la barrera para el estudio que implica el trabajo. Por otro lado, en el caso de los estudiantes que se orientan esencialmente por motivos intrínsecos, el propio disfrute por el estudio o la contribución a la mejora social serían suficientes incentivos para obtener su motivación, independientemente de las horas dedicadas al trabajo remunerado. Ahora bien, resulta interesante observar cómo los mayores niveles de motivación (tanto intrínseca como extrínseca) se observan en aquellos estudiantes que trabajan más de 30 horas semanales, lo que sugiere que, aunque dedicar un elevado número de horas al trabajo representa una evidente barrera para el estudio, una alta motivación, sea del tipo que sea, constituye un motor que puede impedir el desistimiento académico, u otras adversidades con las que se puedan encontrar a lo largo de su itinerario formativo (Figuera y Torrado, 2015). Además, nuestros resultados sugieren que, de forma contraria a los resultados reportados por otras investigaciones (Carmina y Salvador, 2007; García-Garduño y Organista, 2006), la elección de estudios de Educación se basó principalmente en motivos de tipo intrínseco. Estos resultados van en la línea de los estudios de Sultana y Wirtz (1992), y Yong (1995), que relacionan la elección de la carrera académica con el motivo de logro (McClelland, 1961), consistente en la satisfacción de la propia vocación, el disfrute con el aprendizaje en sí mismo, y el desarrollo de las propias competencias y aptitudes, seguidos por la mejora social en general y del entorno más cercano, en consonancia con nuestros resultados, y con los postulados de la teoría de la acción razonada de Deci y Ryan (2000), que sostiene que la autodeterminación es clave a la hora de desarrollar mayores niveles de motivación intrínseca.

Pero, ¿de qué depende, y cómo se construye esta motivación? Los resultados del modelo de regresión múltiple revelaron que el hecho de tener que trabajar durante los estudios afecta a ambos tipos de motivación, especialmente a la extrínseca, sobre todo en el caso de trabajar más de 30 horas semanales. Otras de las variables predictoras de la motivación fueron las relacionadas con el nivel educativo y laboral de los progenitores. Es decir, por lo general, un menor nivel socioeducativo y laboral de los padres predice una mayor motivación, tanto intrínseca como extrínseca, de acuerdo 
con Fachelli y Torrents (2015) o Troiano et al. (2017). No obstante, nuestro modelo obtuvo un valor predictivo del $20 \%$ de la varianza total obtenida en ambos tipos de motivación, por lo que resulta necesario continuar profundizando en los determinantes de la motivación de los estudiantes, tal vez integrando la perspectiva de otras disciplinas afines a la Pedagogía, como, por ejemplo, incorporando el peso de los rasgos de personalidad en la motivación académica, desde la perspectiva de la Psicología de la Educación.

Por otra parte, la satisfacción general con la experiencia universitaria fue elevada en el conjunto de la muestra. En cuanto a la asociación entre los tipos de motivación y la satisfacción, se observan diferencias en las puntuaciones en las diferentes variables de satisfacción académica evaluadas en aquellos estudiantes con mayores niveles de motivación intrínseca y en aquellos con una mayor motivación extrínseca. Nuestros resultados sugieren que la motivación intrínseca se relaciona con una mayor satisfacción con la experiencia universitaria a nivel global. Es decir, aquellas personas que acceden a cursar la carrera por motivos vinculados a cuestiones de satisfacción personal, también estarán más satisfechas con su experiencia como universitario/a (Bandura, 1997; Figuera et al., 2018; Lent et al., 2016). Esto se apoya en que los estudiantes orientados por este tipo de metas puntuaron de forma significativamente superior en aquellas variables relacionadas con el crecimiento como estudiante y futuro profesional de la Educación, siendo estas: 1) satisfacción con los contenidos de los programas formativos, 2) orientación universitaria, 3) relación con del profesorado y, 4) los compañeros (Griffioen et al., 2018; Maurer y Chapman, 2017). Por su parte, los estudiantes orientados por motivos extrínsecos valoraban en mayor medida únicamente el indicador que se podría considerar el más instrumental: los servicios de administración académica, de acuerdo con los resultados aportados por Dorio (2017). En cuanto a la relación entre motivación y satisfacción académica a lo largo del itinerario formativo, si bien los análisis de correlación sugieren una asociación moderada y significativa entre ambas variables, nuestro estudio fue de corte transversal, lo que impide establecer relaciones de causalidad entre motivación, satisfacción con la experiencia universitaria y veteranía en el programa formativo. Por tanto, resulta pertinente realizar en el futuro estudios longitudinales en los que se evalúen ambos parámetros, desde el acceso de los estudiantes a los programas formativos hasta su finalización, para poder esclarecer tal tipo de relaciones de causalidad. Por otra parte, habiendo observado cómo la satisfacción con la experiencia académica decae (levemente, pero de forma significativa) en los últimos cursos de los programas formativos, y dada la relación existente entre motivación intrínseca y un mejor desempeño laboral y académico señalada entre otros, por Avendaño-Bravo y González-Urrutia (2012), también resulta prioritario establecer en el ámbito universitario mecanismos y estrategias que 
promuevan este tipo de motivos, para fomentar un mejor desempeño laboral futuro de los profesionales de la Educación. Dentro de este tipo de medidas orientadas a aumentar la motivación intrínseca podrían incluirse aquellas que fomenten la autodeterminación y la participación de los estudiantes en su propio itinerario académico, y que se orienten específicamente a satisfacer los motivos de logro y afiliación. Por tanto, se podrían incluir estrategias como fomentar la libre elección de las materias a través de una mayor oferta de asignaturas optativas, la creación de menciones o itinerarios adaptados a las inquietudes académicas de los estudiantes, la mentoría de los estudiantes noveles por parte de estudiantes de cursos superiores, o la oferta de prácticas extracurriculares en entidades educativas del entorno, o de programas de Aprendizaje-Servicio, que conecten la universidad con su entorno cercano.

Finalmente, a pesar del elevado número de participantes en el estudio, nuestros resultados deben ser tomados con cautela a nivel de comparativa internacional, debido a la diferencia entre el número de respuestas obtenidas de América Latina y de Europa, siendo muy superior el volumen de participantes de instituciones latinoamericanas. Además, es necesario considerar como posible limitación de nuestra investigación la diversidad de contextos, culturas y sistemas educativos comparados en este trabajo, en relación con la posible generalización de nuestros resultados. Para estudios futuros, se considera especialmente pertinente profundizar en la influencia del género sobre la motivación académica, en el papel de la motivación sobre la participación académica, así como sobre la participación misma de determinadas minorías (estudiantes con alguna situación de diversidad funcional, o alumnos y alumnas pertenecientes a pueblos indígenas) en la vida universitaria.

\section{NOTAS}

1 Este trabajo se enmarca en el Proyecto From Tradition to Innovation in Teacher-Training Institutions (TO INN), 573685-EPP-2016-1-ES-EPP KA2-CBHE-JP, financiado por la Unión Europea a través del programa Erasmus + Key Action 2 Capacity Building. Coordinado por la Dra. Alejandra Montané de la Universidad de Barcelona en la que participan 21 instituciones de Educación Superior de ocho países de América Latina (Colombia, Argentina, Honduras, México, Ecuador, Brasil, Bolivia y Paraguay) y cinco países de la UE (España, Italia, Holanda, Francia y Portugal). 


\section{REFERENCIAS BIBLIOGRÁFICAS}

Abarca, N., Gormaz, N., y Leiva, P.I. (2012). Expectativas de roles futuros de estudiantes universitarios en Chile. Universitas Psychologica, 11(2), 405-414. https://doi.org/10.11144/ Javeriana.upsy11-2.erfe

Ariño, A. i Sintes, E. (2016). Via Universitària: Ser estudiant universitari avui. Fundació Jaume Bofill.

Avendaño-Bravo, C. y GonzálezUrrutia, R. (2012). Motivos para ingresar a las carreras de Pedagogía de los estudiantes de primer año de la Universidad de Concepción. Estudios Pedagógicos, 2, 21-33. http://dx.doi.org/10.4067/S071807052012000200002

Bandura, A. (1997). Self-efficacy. The exercise of control. Freeman.

Cabrera, A.F., Pérez, P., y López, L. (2014). Evolución de las perspectivas de estudio de la retención universitaria en los EE.UU.: Bases conceptuales y puntos de inflexión. En P. Figuera (Coord.), Persistir con éxito en la universidad: De la investigación a la acción (pp. 15-40), Laertes.

Chandra, T., Ng, M., Chandra, S., \& Priyono (2018). The Effect of Service Quality on Student Satisfaction and Student Loyalty: An Empirical Study. Journal of Social Studies Education Research, 9(3), 109-131.

Camina, A. y Salvador, M.I. (2007). Condicionantes y características de los estudiantes que inician magisterio. Estudio descriptivo y comparativo entre especialidades. Tendencias pedagógicas, 12, 245-262.

Cano-Celestino. M.A. (2008). Motivación y elección de carrera. Revista Mexicana de Orientación Educativa, 5(13), 6-9.
Deci, E.L. \& Ryan, R.M.(2000). The "what" and "why" of goal pursuits: Human needs and the self-determination of behaviour. Psychological Inquiry, 11, 227-268. https://doi.org/10.1207/ S15327965PLI1104_01

Dorio, I. (2017). La transición a la Universidad. El grado de maestro de Educación Infantil [Tesis doctoral], Universidad de Barcelona. http://hdl. handle.net/2445/109484

Figuera, P., Buxarrais, M.R., Llanes, J., y Venceslao, M. (2018). Perfil, motivación y satisfacción académica en los estudiantes de máster: el caso de Ciencias Sociales y Jurídicas. Estudios sobre Educación, 34, 219-237. http:// dx.doi.org/10.15581/004.34.219-237

Figuera, P. y Torrado, M. (2015). The transition to university of atrisk groups in Spain: the case of students from vocational education and training. Revista de Cercetare şi Interventie Socială, 49, 23-40.

Figuera, P., Torrado, M., Dorio, I., y Freixa, I. (2015). Trayectorias de persistencia y abandono de estudiantes universitarios no convencionales: implicaciones para la orientación. Revista Electrónica Interuniversitaria de Formación del Profesorado, 18(2), 1-18. https://doi.org/10.6018/ reifop.18.2.220101

Gámez, E., Marrero, H., Díaz, J.M., y Urrutia, M. (2015). ¿Qué esperan encontrar los alumnos en los estudios de Psicología? Metas y motivos personales de los estudiantes en su primer año en la universidad. Anales de Psicología, 31(2), 589-599. https:// doi.org/10.6018/analesps.31.2.171851

Gámez, E. y Marrero, H. (2003). Metas y motivos en la elección de la carrera 
universitaria: Un estudio comparativo entre psicología, derecho y biología. Anales de Psicología, 19(1), 121-131.

García-Garduño, J.M. y Organista, J. (2006). Motivación y expectativas para ingresar a la carrera de profesor de educación primaria: Un estudio de tres generaciones de estudiantes normalistas mexicanos de primer ingreso. Revista Electrónica de Investigación Educativa, 8(2).

García-Ripa, M.I., Sánchez-García, M.F., y Risquez, A. (2017). Perfiles motivacionales de elección de estudios en estudiantes universitarios de nuevo ingreso. Univesitas Psychologica, 17(3). 1-12. https://doi.org/10.11144/ Javeriana.upsy17-3.pmee

Griffioen, D.M.E., Doppenberg, J.J., \& Oostdam, R.J. (2018). Are more able students in higher education less easy to satisfy? Higher Education, 75, 891907. https://doi.org/10.1007/s10734017-0176-3

Martínez-García, J.S. (2017). Sobrecualificación de los titulados universitarios y movilidad social. Papers. Revista de Sociología, 102(1), 29-52. http://dx.doi.org/10.5565/rev/ papers. 2225

Maurer, T.J. \& Chapman, E.F. (2017). Relationship of Proactive Personality with Life Satisfaction during Late Career and Early Retirement. Journal of Career Development, 1-16. https:// doi.org/10.1177/0894845317697381

McClelland, D.C. (1961). The Achieving Society. Van Nostrand.

Méndez-Ulrich, J.L., Montané, A., Llanes, J., Hervás, G., Calduch, I., Morales-Ulloa, R., y Muñoz, J. (2019). Características sociodemográficas y condiciones de vida y estudio de los estudiantes de Educación de origen indígena. La aproximación del proyecto TO-INN. Revista de Sociología de la Educación, 2(2), 131-148. http:// dx.doi.org/10.7203/RASE.12.2.14810

Montané. A., Llanes, J., Calduch, I., Hervás, G., Méndez-Ulrich, J.L., \& Muñoz. J. (2019). The social dimension in Higher Education. Design and implementation of an instrument for student analytics in a Latin American context. In A. Bon, M. Pini, M. \& H. Akkermans (Coords.), Culture, citizenship, participation. Comparative perspectives from Latin America on inclusive Education (pp. 133-158). Pangea. https://bit.ly/3h5QZS2

Lent, R.W., Taveira M., Figuera, P., Dorio, I., Faria S., \& Gonçalves, A.M. (2016). Test of the Social Cognitive Model of Well-Being in Spanish College Students. Journal of Career Assessment, 25(1), 135-143. https://doi. org/10.1177/1069072716657821

Skatova, A. \& Ferguson, E. (2014). Why do different people choose different university degrees? Motivation and the choice of degree. Frontiers in Psychology, 5, 1-15. https://doi. org/10.3389/fpsyg.2014.01244

Sultana, Q. \& Wirtz, P. (1992). A comparative study of 1985 and 1991 teacher education candidates. The Twenty First Annual Conference of Mid-South Educational Research Association.

Tinto, V. (2017). Through the eyes of students. Journal of College student retention: Research, Theory \& Practice, 19(3), 254-269. https://doi. org/10.1177/1521025115621917

Torrents, D. y Fachelli, S. (2015). El efecto del origen social con el paso del tiempo: la inserción laboral de los graduados universitarios españoles durante la democracia. Revista Complutense de Educación, 26(2), 
331-349. http://dx.doi.org/10.5209/rev_ RCED.2015.v26.n2.43070

Troiano, H., Torrents, D., SánchezGelabert, A., y Daza, L. (2017). Evolución del acceso a la universidad y de la elección de titulación universitaria entre la población joven de Catalunya. Cuadernos de Relaciones Laborales, 35(2), 281-303. http//doi. org/10.5209/CRLA.56775

Vázquez-Alonso, A. y Manassero-Mas, M.A. (2015). La elección de estudios superiores científico-técnicos: análisis de algunos factores determinantes en seis países. Revista Eureka sobre Enseñanza y Divulgación de las
Ciencias, 12 (2), 264-277. http://dx.doi. org/10.25267/Rev_Eureka_ensen_ divulg_cienc.2015.v12.i2.03

Weerasinghe, I.M.S., Lalitha, R., \& Fernando, S. (2018). University facilities and student satisfaction in Sri Lanka. International Journal of Educational Management, 32(5), 866880. https://doi.org/10.1108/IJEM-072017-0174

Yong, S.Y. (1995). Teacher trainees' motives for entering into a teaching career in Brunei Darussalam. Teaching and Teacher Education, 11, 275280. https://doi.org/10.1016/0742051X(94)00023-Y 


\section{PERFIL ACADÉMICO Y PROFESIONAL DE LOS AUTORES}

Juan Llanes Ordóñez. ORCID: http://orcid.org/0000-0002-0059-9741

Profesor lector Departamento de Métodos de Inverstigación y Diagnóstico en Educación de la Universidad de Barcelona. Líneas de investigación: la orientación e inserción profesional de estudiantes jóvenes y adultos, la gestión de la carrera y empleabilidad de las personas en la educación superior y la motivación y satisfacción de los estudiantes con la educación. E-mail: juanllanes@ub.edu 0607

Jorge Luis Méndez-Ulrich. ORCID: http://orcid.org/0000-0001-9718-

Profesor lector del Departamento de Métodos de Inverstigación y Diagnóstico en Educación de la Universidad de Barcelona. Grupo de Investigación en Intervenciones Socioeducativas en la Infancia y la Juventud (GRISIJ). Líneas de investigación: Educación inclusiva, educación superior, Educación para la Salud. E-mail: jordi.mendez@ub.edu 8076

Alejandra Montané López. ORCID: http://orcid.org/0000-0002-3844-

Profesora agregada del Departamento de Didáctica y Organización Educativa de la Universidad de Barcelona. Líneas de investigación: políticas de educación superior y formación del profesorado. E-mail: smontane@ ub.edu

Fecha Recepción del Artículo: 21. Enero. 2020

Fecha Modificación del Artículo: 01. Junio. 2020

Fecha Aceptación del Artículo: 11. Junio. 2020

Fecha Revisión para Publicación: 15. Junio. 2020 